KYUNGPOOK Math. J. 52(2012), 245-264

http://dx.doi.org/10.5666/KMJ.2012.52.3.245

\title{
Lens Surgeries along the $n$-twisted Whitehead Link
}

\author{
Teruhisa KadoKami* \\ Department of Mathematics, East China Normal University, Dongchuan-lu 500, \\ Shanghai 200241, China \\ e-mail: mshj@math.ecnu.edu.cn, kadokami2007@yahoo.co.jp \\ NORIKO MARUYAMA \\ Musashino Art University, Ogawa 1-736, Kodaira, Tokyo 187-8505, Japan \\ e-mail : maruyama@musabi.ac.jp \\ Masafumi ShimozaWA \\ Department of Mathematics, Tokyo Woman's Christian University, Zempukuji 2-6- \\ 1, Suginami-ku, Tokyo 167-8585, Japan \\ e-mail : mas230jcom.home.ne.jp
}

Dedicated to Professor Akio Kawauchi for his 60th birthday.

Abstract. We determine lens surgeries (i.e. Dehn surgery yielding a lens space) along the $n$-twisted Whitehead link. To do so, we first give necessary conditions to yield a lens space from the Alexander polynomial of the link as: (1) $n=1$ (i.e. the Whitehead link), and (2) one of surgery coefficients is 1, 2 or 3 . Our interests are not only lens surgery itself but also how to apply the Alexander polynomial for this kind of problems.

\section{Introduction}

For a $\mu$-component link $L=K_{1} \cup \ldots \cup K_{\mu}$ in an integral homology 3-sphere $\Sigma$, Dehn surgery is an operation to $\Sigma$ by attaching solid tori to the boundaries of the exterior of $L$, where the way to attach a solid torus is parametrized by a rational number or $1 / 0=\infty$ or $\emptyset$. The parameter is called a surgery coefficient. The result of $\left(r_{1}, \ldots, r_{\mu}\right)$-surgery along $L$ is obtained by Dehn surgery along $K_{i}$ with a surgery coefficient $r_{i} \in \mathbb{Q} \cup\{\infty, \emptyset\}$ for every $i=1, \ldots, \mu$. We say that Dehn surgery is a lens surgery if the resulting space is a lens space. Let $W_{n}=K_{1} \cup K_{2}(n \in \mathbb{Z})$ be the $n$-twisted Whitehead link as in Figure 1, where a rectangle with an integer $m$

* Corresponding Author.

Received February 20, 2010; Revised May 9, 2011;accepted November 23, 2011.

2010 Mathematics Subject Classification: 57M25, 57M27, 57Q10.

Key words and phrases: Dehn surgery, lens space, Reidemeister torsion, Alexander polynomial, Rolfsen move. 
implies a righthand $m$-full twists if $m \geq 0$, or a lefthand $|m|$-full twists if $m<0$. In the present paper, we determine when Dehn surgery along $W_{n}$ yields a lens space by using the Reidemeister torsion and Rolfsen moves.

In the present paper, we are mainly concerned with the restriction on the Alexander polynomial of a link to admit a lens surgery. Our interests are not only lens surgery itself but also how to apply the Alexander polynomial for this kind of problems. For examples: (i) The first author [8] gave necessary conditions on the Alexander polynomial of an algebraically split component-preservingly amphicheiral link. Consideration on the $\operatorname{sign} \varepsilon_{n}$ in Theorem 1.1. (relation with chirality of the links) motivates the work (see Remark 6.4.). (ii) The first author [9] determined lens surgeries along the Milnor links, and clarified that we cannot obtain the result by only the Alexander polynomial. Our method extends to algebraically same links with $W_{n}$ (see Section 6).

L. Moser [17] determined Dehn surgery along every torus knot by the Seifert fibered structure of the exterior. Recently, the first author and the third author [10] determined Dehn surgery along every torus link by essentially the same method. R. Fintushel and R. J. Stern [3], and the second author [16] gave examples of hyperbolic knots yielding lens spaces. Moreover the second author [16] pointed out that a 2-bridge link $C(m, m)$ where $m$ is odd in Conway's notation (cf. [13, Section 2]) can yield a lens space. Note that $W_{n}$ is also a 2 -bridge link $C(2,2 n,-2)$. J. Berge [1] showed that a doubly primitive knot yields a lens space. It is conjectured that a knot in $S^{3}$ yielding a lens space is a doubly primitive knot. Ordinarily, when we study lens surgeries along a knot or a link, we use a geometric structure of the complement of it [15], and apply Cyclic Surgery Theorem [2] or knot Floer homology [18] or more geometric cut and paste arguments [4].

Let $M=\left(W_{n} ; p_{1} / q_{1}, p_{2} / q_{2}\right)$ denote the result of $\left(p_{1} / q_{1}, p_{2} / q_{2}\right)$-surgery along $W_{n}$. Since the linking number of $W_{n}$ is zero, the first homology $H_{1}(M)$ is finite cyclic if and only if $\operatorname{gcd}\left(p_{1}, p_{2}\right)=1$ and $p_{1} p_{2} \neq 0$, and the order of $H_{1}(M)$ is $p=\left|p_{1} p_{2}\right|$. We note that $W_{0}$ is the 2-component trivial link, $W_{ \pm 1}$ is the Whitehead link, and $W_{-n}$ is the mirror image of $W_{n}$. Hence it is sufficient to consider the case $n>0$. Thus we fix the following setting.

Setting (1) $W_{n}=K_{1} \cup K_{2}$ is the 2-component link in $S^{3}$ of Figure 1, where $n>0$.

(2) $M=\left(W_{n} ; p_{1} / q_{1}, p_{2} / q_{2}\right)$ is the result of $\left(p_{1} / q_{1}, p_{2} / q_{2}\right)$-surgery along $W_{n}$, where $q_{i} \geq 1(i=1,2), \operatorname{gcd}\left(p_{1}, p_{2}\right)=1$ and $p=\left|p_{1} p_{2}\right| \geq 2$.

Throughout this paper, $\zeta_{d}$ is a primitive $d$-th root of unity and $\mathbb{Q}\left(\zeta_{d}\right)$ is the $d$-th cyclotomic field, for an integer $d \geq 2$.

Let $M$ be a homology lens space with $H_{1}(M) \cong \mathbb{Z} / p \mathbb{Z}(p \geq 2)$, and $T$ a generator of $H_{1}(M)$. Let $d \geq 2$ be a divisor of $p$, and $\psi: \mathbb{Z}\left[H_{1}(M)\right] \rightarrow \mathbb{Q}\left(\zeta_{d}\right)$ a ring homomorphism such that $\psi(T)=\zeta_{d}$. Then $\tau^{\psi}(M) \in \mathbb{Q}\left(\zeta_{d}\right)$, the Reidemeister torsion of $M$ associated to $\psi$, is determined up to multiplications by $\pm \zeta_{d}^{m}(m \in \mathbb{Z})$ 


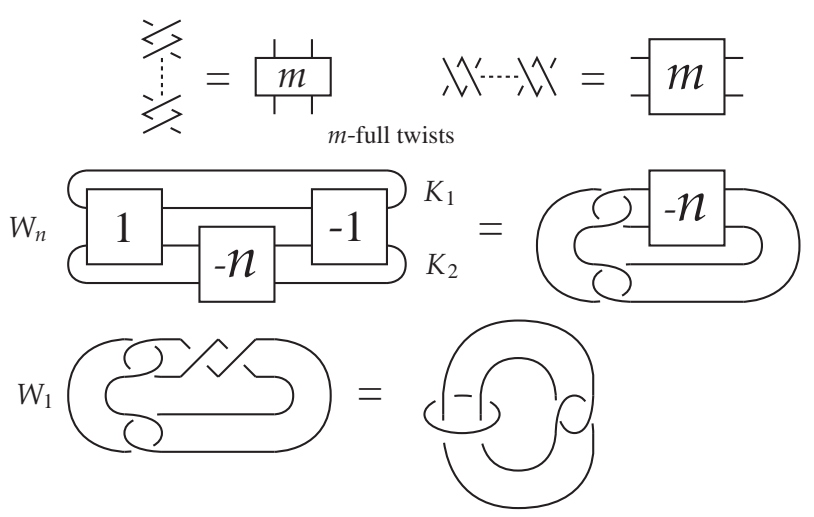

Figure 1: $n$-twisted Whitehead link $W_{n}$

(see $[22,23]$ for details on the Reidemeister torsion). For $A$ and $B$ in $\mathbb{Q}\left(\zeta_{d}\right)$, if there exists an integer $m$ such that $A= \pm \zeta_{d}^{m} B$, then we denote by $A \doteq B$.

We first state a key theorem of the present paper.

Theorem 1.1. Let $M=\left(W_{n} ; p_{1} / q_{1}, p_{2} / q_{2}\right)$ be as in the setting above. Then we have the following:

(1) Let $d \geq 2$ be a divisor of $p_{2}$, and $\psi: \mathbb{Z}\left[H_{1}(M)\right] \rightarrow \mathbb{Q}\left(\zeta_{d}\right)$ a ring homomorphism defined by $\psi\left(\left[m_{1}\right]\right)=1$ and $\psi\left(\left[m_{2}\right]\right)=\zeta_{d}$, where $m_{i}$ is a meridian of $K_{i}$. Then we have

$$
\tau^{\psi}(M) \doteq\left\{n q_{1}\left(\zeta_{d}-1\right)^{2}+\varepsilon_{n} p_{1} \zeta_{d}\right\}\left(\zeta_{d}-1\right)^{-1}\left(\zeta_{d}^{\bar{q}_{2}}-1\right)^{-1},
$$

where $\varepsilon_{n}=1$ or -1 , and $q_{2} \bar{q}_{2} \equiv 1\left(\bmod p_{2}\right)$.

(2) Let $d \geq 2$ be a divisor of $p_{1}$, and $\psi: \mathbb{Z}\left[H_{1}(M)\right] \rightarrow \mathbb{Q}\left(\zeta_{d}\right)$ a ring homomorphism defined by $\psi\left(\left[m_{1}\right]\right)=\zeta_{d}$ and $\psi\left(\left[m_{2}\right]\right)=1$, where $m_{i}$ is a meridian of $K_{i}$. Then we have

$$
\tau^{\psi}(M) \doteq\left\{n q_{2}\left(\zeta_{d}-1\right)^{2}+\varepsilon_{n} p_{2} \zeta_{d}\right\}\left(\zeta_{d}-1\right)^{-1}\left(\zeta_{d}^{\bar{q}_{1}}-1\right)^{-1},
$$

where $\varepsilon_{n}=1$ or -1 , and $q_{1} \bar{q}_{1} \equiv 1\left(\bmod p_{1}\right)$.

(3) In (1) and (2), we have $\varepsilon_{1}=1$.

We have two remarks on the proof of Theorem 1.1.. (i) Since $W_{n}$ is an interchangeable link (i.e. as an ordered link, $K_{1} \cup K_{2}$ is ambient isotopic to an ordered link $K_{2} \cup K_{1}$ ), it is sufficient to show Theorem 1.1. (1). We will often omit a half of the proofs by the same reason (ex. Theorem 1.2., Lemma 4.1. and Lemma 4.2.). (ii) 
To show Theorem 1.1., we applied the surgery formula of the Reidemeister torsion due to V. G. Turaev [22, 23] (cf. Lemma 2.1.).

Let $L(p, q)$ be a lens space which is defined as the result of $p / q$-surgery along the trivial knot. By comparing the Reidemeister torsion of $M$ as in Theorem 1.1. and that of $L(p, q)$ (in Example 2.2.), we have:

Theorem 1.2. Let $M=\left(W_{n} ; p_{1} / q_{1}, p_{2} / q_{2}\right)$ be as in the setting above. Then we have the following:

(A) If $M$ is a lens space, then we have $n=1$.

(B) The resulting space $M=\left(W_{1} ; p_{1} / q_{1}, p_{2} / q_{2}\right)$ is a lens space if and only if one of the following (1), (2), (3), (4), (5) or (6) holds:

(1) $p_{1} / q_{1}=1$ and $\left|p_{2}-6 q_{2}\right|=1$.

(2) $p_{1} / q_{1}=2$ and $\left|p_{2}-4 q_{2}\right|=1$.

(3) $p_{1} / q_{1}=3$ and $\left|p_{2}-3 q_{2}\right|=1$.

(4) $p_{2} / q_{2}=1$ and $\left|p_{1}-6 q_{1}\right|=1$.

(5) $p_{2} / q_{2}=2$ and $\left|p_{1}-4 q_{1}\right|=1$.

(6) $p_{2} / q_{2}=3$ and $\left|p_{1}-3 q_{1}\right|=1$.

Moreover if (1), (2), (3), (4), (5) or (6) holds, then $M=L\left(p_{2}, 4 q_{2}\right), L\left(2 p_{2}, 8 q_{2}\right.$ $\left.-p_{2}\right), L\left(3 p_{2}, 3 q_{2}-2 p_{2}\right), L\left(p_{1}, 4 q_{1}\right), L\left(2 p_{1}, 8 q_{1}-p_{1}\right)$ or $L\left(3 p_{1}, 3 q_{1}-2 p_{1}\right)$, respectively.

We remark that six cases in Theorem 1.2. are not exclusive, for example $\left(p_{1} / q_{1}, p_{2} / q_{2}\right)=(2,3)$ in $(2)$ and $(6)$, and $\left(p_{1} / q_{1}, p_{2} / q_{2}\right)=(3,2)$ in (3) and (5).

B. Martelli and C. Petronio [15] completely determined exceptional Dehn fillings of the complement of the chain link with three components by using hyperbolic geometry. The complement of $W_{-1}$ is a certain Dehn filling of the 3-component chain link. Though their result overlaps with Theorem 1.2, the overlap is only partial, our method is different from theirs, and our targets are extended (i.e. our results are 'not' properly included in theirs).

In Section 2, we provide basic tools of this paper such as Reidemeister torsion and Rolfsen moves. In Section 3, we prove Theorem 1.1. In Section 4, we prove "only if part" of Theorem 1.2. by using Theorem 1.1. In Section 5, we prove "if part" of Theorem 1.2. by using Rolfsen moves. In Section 6, we will apply our method for a 2-component link and its components with the same Alexander polynomials as $W_{n}$.

We refer to $[5,6,7,9,11,12]$ for studies on Dehn surgery by using the Reidemeister torsion. 


\section{Preliminaries}

\subsection{Reidemeister torsion}

We rewrite a surgery formula due to Turaev to be suitable for the present paper. For details, see $[22,23]$, and see also [6, Section 2].

Let $R$ be a commutative ring with nonzero identity element. Then we denote the classical ring of quotient by $Q(R)$. Let $X$ be a finite CW complex. Then the maximal abelian torsion of $X$, denoted by $\tau(X)$, is an element of $Q\left(\mathbb{Z}\left[H_{1}(X)\right]\right)$ that is determined up to multiplication by an element of $\pm H_{1}(X)$, which is defined from a chain complex $\mathbf{C}_{*}$ induced by the maximal abelian covering of $X$.

Let $L=K_{1} \cup \cdots \cup K_{\mu}$ be an oriented $\mu$-component link in an integral homology 3 -sphere $\Sigma$, and $\Delta_{L}\left(t_{1}, \ldots, t_{\mu}\right)$ the Alexander polynomial of $L$, where a variable $t_{i}$ is represented by a meridian of $K_{i}(i=1, \ldots, \mu)$. We note that if the orientation of $K_{i}$ is reversed, then the variable $t_{i}$ is replaced with $t_{i}^{-1}$, and that the set of the $\mu$-variable Alexander polynomials of $\mu$-component links in $S^{3}$ coincides with the set of the $\mu$-variable Alexander polynomials of $\mu$-component links in any homology 3 -sphere $\Sigma$.

Let $L=K_{1} \cup K_{2} \cup K_{3}$ be a 3-component link in an integral homology 3-sphere $\Sigma, E_{L}$ the exterior of $L, m_{i}$ and $l_{i}$ a meridian and a longitude of $K_{i}(i=1,2,3)$ on $\partial E_{L}$ respectively. Let $M=\left(L ; p_{1} / q_{1}, p_{2} / q_{2}, p_{3} / q_{3}\right)$ be the result of $p_{i} / q_{i}$-surgery along $K_{i}$, and set

$$
M=E_{L} \cup V_{1} \cup V_{2} \cup V_{3} \text { and } M_{0}=E_{L} \cup V_{1} \cup V_{2} \text {, }
$$

where $V_{i}$ is a solid torus glued in doing surgery along $K_{i}$. Let $l_{i}^{\prime}$ be the core of $V_{i}$. Note that the homology class of $l_{i}^{\prime}$ is uniquely determined in $M_{0}(i=1,2)$ and in $M$ $(i=3)$. We assume that $M$ is a homology lens space with $H_{1}(M) \cong \mathbb{Z} / p \mathbb{Z}(p \geq 2)$. Let $T$ be a generator of $H_{1}(M), d \geq 2$ a divisor of $p$ and $\psi: \mathbb{Z}\left[H_{1}(M)\right] \rightarrow \mathbb{Q}\left(\zeta_{d}\right)$ a ring homomorphism such that $\psi(T)=\zeta_{d}$. We define $\psi_{0}: \mathbb{Z}\left[H_{1}\left(M_{0}\right)\right] \rightarrow \mathbb{Q}\left(\zeta_{d}\right)$ by $\psi_{0}=\psi \circ \iota$ where $\iota: \mathbb{Z}\left[H_{1}\left(M_{0}\right)\right] \rightarrow \mathbb{Z}\left[H_{1}(M)\right]$ is a ring homomorphism induced from the natural inclusion $M_{0} \hookrightarrow M$. Then we have the following surgery formula for the Reidemeister torsion.

Lemma 2.1(surgery formula; Turaev [22, 23]).

(1) If $\left[l_{i}^{\prime}\right](i=1,2)$ has infinite order in $H_{1}\left(M_{0}\right)$, then we have

$$
\tau\left(M_{0}\right) \doteq \Delta_{L}\left(\left[m_{1}\right],\left[m_{2}\right],\left[m_{3}\right]\right)\left(\left[l_{1}^{\prime}\right]-1\right)^{-1}\left(\left[l_{2}^{\prime}\right]-1\right)^{-1} \quad \text { in } Q\left(\mathbb{Z}\left[H_{1}\left(M_{0}\right)\right]\right) .
$$

(2) If $\tau\left(M_{0}\right) \neq 0$ and $\psi\left(\left[l_{3}^{\prime}\right]\right) \neq 1$, then we have

$$
\tau^{\psi}(M) \doteq \psi_{0}\left(\tau\left(M_{0}\right)\right)\left(\psi\left(\left[l_{3}^{\prime}\right]\right)-1\right)^{-1} .
$$


Example 2.2(Reidemeister [19]). Let $T$ be a generator of $H_{1}(L(p, q))$. Let $d \geq 2$ be a divisor of $p$, and $\psi: \mathbb{Z}\left[H_{1}(L(p, q))\right] \rightarrow \mathbb{Q}\left(\zeta_{d}\right)$ a ring homomorphism such that $\psi(T)=\zeta_{d}$. Then we have

$$
\tau^{\psi}(L(p, q)) \doteq\left(\zeta_{d}^{i}-1\right)^{-1}\left(\zeta_{d}^{i \bar{q}}-1\right)^{-1}
$$

for some $i$ where $\operatorname{gcd}(i, d)=1$ and $q \bar{q} \equiv 1(\bmod d)$.

Lemma 2.3(Torres formula [21]). Let $L=K_{1} \cup \cdots \cup K_{\mu} \cup K_{\mu+1}(\mu \geq 1)$ be an oriented $(\mu+1)$-component link in an integral homology 3-sphere $\Sigma$ and $L^{\prime}=$ $K_{1} \cup \cdots \cup K_{\mu}$ a $\mu$-component sublink. Then we have

$$
\Delta_{L}\left(t_{1}, \ldots, t_{\mu}, 1\right) \doteq\left\{\begin{array}{cc}
\frac{t^{\ell}-1}{t-1} \Delta_{K}(t) & (\mu=1), \\
\left(t_{1}^{\ell_{1}} \cdots t_{\mu}^{\ell_{\mu}}-1\right) \Delta_{L^{\prime}}\left(t_{1}, \ldots, t_{\mu}\right) & (\mu \geq 2),
\end{array}\right.
$$

where $\ell_{i}=\operatorname{lk}\left(K_{i}, K_{\mu+1}\right)(i=1, \ldots, \mu)$ is the linking number of $K_{i}$ and $K_{\mu+1}$, and we set $L=K_{1}=K, t=t_{1}$ and $\ell=\ell_{1}$ if $\mu=1$.

Lemma 2.4(duality; Turaev [22]). Let $L=K_{1} \cup \cdots \cup K_{\mu}$ be an oriented $\mu$ component link in an integral homology 3 -sphere $\Sigma$. We set $\ell_{i j}$ is the linking number of $K_{i}$ and $K_{j}(1 \leq i \neq j \leq \mu)$ if $\mu \geq 2$, and $L=K_{1}=K$ and $t=t_{1}$ if $\mu=1$. Then we have the following:

$$
\begin{array}{cc}
\Delta_{K}(t)=t^{a} \Delta_{K}\left(t^{-1}\right) & (\mu=1), \\
\Delta_{L}\left(t_{1}, t_{2}, \ldots, t_{\mu}\right)=(-1)^{\mu} t_{1}^{a_{1}} t_{2}^{a_{2}} \cdots t_{\mu}^{a_{\mu}} \Delta_{L}\left(t_{1}^{-1}, t_{2}^{-1}, \ldots, t_{\mu}^{-1}\right) & (\mu \geq 2),
\end{array}
$$

where $a$ is even and $a_{i} \equiv 1+\sum_{j \neq i} \ell_{i j}(\bmod 2)$.

Remark 2.5. Torres [21] has already shown a duality of the Alexander polynomials. Lemma 2.4. is a refinement of the duality.

The following lemma is used effectively to prove "only if part" of Theorem 1.2. in Section 4.

Lemma 2.6. Let $\ell \geq 5$ be a prime. Suppose that two Laurent polynomials $F(t)$ and $G(t) \in \mathbb{Z}\left[t, t^{-1}\right]$ are of the form:

$$
\begin{aligned}
& F(t)=a_{0}+\sum_{i=1}^{\frac{\ell-3}{2}} a_{i}\left(t^{i}+t^{-i}\right) \\
& G(t)=b_{0}+\sum_{i=1}^{\frac{\ell-3}{2}} b_{i}\left(t^{i}+t^{-i}\right)
\end{aligned} \quad\left(a_{i}, b_{i} \in \mathbb{Z} ; i=0,1, \ldots, \frac{\ell-3}{2}\right)
$$

and $F\left(\zeta_{\ell}\right)=G\left(\zeta_{\ell}\right)$ holds for any $\ell$-th root of unity $\zeta_{\ell}$. Then we have $F(t)=G(t)$. 
Proof. By the assumption, $F(t)-G(t)$ is divisible by $t^{\ell-1}+t^{\ell-2}+\cdots+t+1$. Since the degree of $F(t)-G(t)$ does not exceed $\ell-3$ by the form, we have $F(t)-G(t) \equiv 0$. $\square$

\subsection{Rolfsen moves}

We recall Rolfsen moves on Dehn surgery. It is known that a pair of Dehn surgeries describes the same 3-manifold if and only if they are moved to each other by Rolfsen moves [20]. Rolfsen move consists of two moves; an (R1)-move and an (R2)-move. Let $L=K_{1} \cup \cdots \cup K_{\mu}$ be a $\mu$-component link, and $M=\left(L ; r_{1}, \ldots, r_{\mu}\right)$ the result of Dehn surgery along $L$.

(R1)-move: When the $i$-th component $K_{i}$ is unknotted, we may operate $u$-full twists along $K_{i}$ where " $u$-full twists" means righthand $u$-full twists if $u \geq 0$, and lefthand $|u|$-full twists if $u<0$. Then $K_{i}, r_{i}, K_{j}(j \neq i)$ and $r_{j}$ change into $K_{i}^{\prime}, r_{i}^{\prime}, K_{j}^{\prime}(j \neq i)$ and $r_{j}^{\prime}$, respectively, where

$$
r_{i}^{\prime}=\frac{1}{u+1 / r_{i}} \quad \text { and } \quad r_{j}^{\prime}=r_{j}+u\left(\operatorname{lk}\left(K_{i}, K_{j}\right)\right)^{2}
$$

$(1 / 0=\infty$ and $1 / \infty=0)$, and $\mathrm{lk}\left(K_{i}, K_{j}\right)$ is the linking number of $K_{i}$ and $K_{j}$. In Figure 2, an (R1)-move from the lefthand side to the righthand side is 1-full twist along $K_{i}$.

(R2)-move: Adding a new component $K_{\mu+1}$ to $L$ with a framing $\infty$, and its inverse.

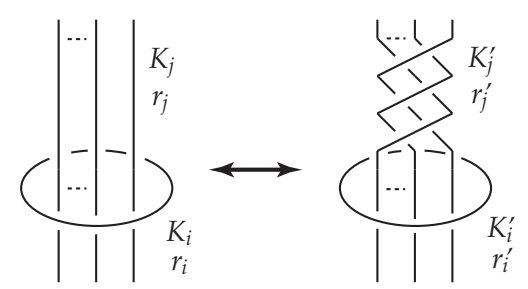

Figure 2: Rolfsen moves

Remark 2.7. R. Fintushel and R. J. Stern [3], and the second author [16] found families of knots yielding a lens space by using another method, called "Kirby moves" [14].

\section{Proof of Theorem 1.1.}

Proof of (1) and (2). We prove only the case (1). Let $d \geq 2$ be a divisor of $p_{2}$. Then $\operatorname{gcd}\left(d, p_{1}\right)=1$.

When we use the surgery formula of the Reidemeister torsion (cf. Lemma 2.1.), not to make the denominator and the numerator vanish, we add the third component 
$K_{3}$ to $W_{n}$ as in Figure 3. Then $H_{i}=K_{i} \cup K_{3}(i=1,2)$ is the Hopf link. We set $\bar{W}=K_{1} \cup K_{2} \cup K_{3}$ and orient $\bar{W}$ so that $\mathrm{lk}\left(K_{i}, K_{3}\right)=1(i=1,2)$. We compute the Reidemeister torison of $M=\left(\bar{W} ; p_{1} / q_{1}, p_{2} / q_{2}, \infty\right)$. Note that the value does not depend on $K_{3}$ because we close up $K_{3}$ by $\infty$-surgery.

By the Torres formula (Lemma 2.3.) and that

$$
\Delta_{W_{n}}\left(t_{1}, t_{2}\right) \doteq n\left(t_{1}-1\right)\left(t_{2}-1\right),
$$

we may set as follows:

$$
\Delta_{\bar{W}}\left(t_{1}, t_{2}, t_{3}\right)=n\left(t_{1} t_{2}-1\right)\left(t_{1}-1\right)\left(t_{2}-1\right)+\left(t_{3}-1\right) g_{n}\left(t_{1}, t_{2}, t_{3}\right)
$$

for some $g_{n}\left(t_{1}, t_{2}, t_{3}\right) \in \mathbb{Z}\left[t_{1}^{ \pm 1}, t_{2}^{ \pm 1}, t_{3}^{ \pm 1}\right]$.

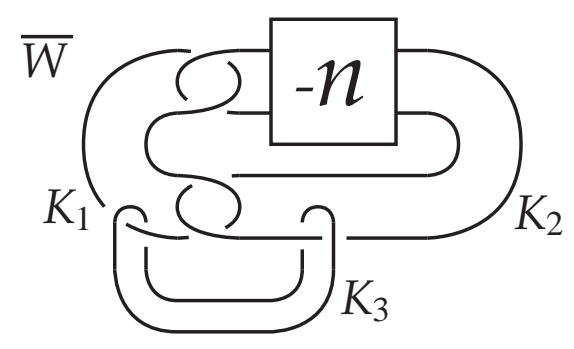

Figure 3: 3-component link $\bar{W}$

Then we have the following lemma:

\section{Lemma 3.1.}

$$
g_{n}\left(t_{1}, 1, t_{3}\right) \doteq 1 \quad \text { and } \quad g_{n}\left(1, t_{2}, t_{3}\right) \doteq 1 .
$$

Proof. By (3.1), we have

$$
\Delta_{\bar{W}}\left(t_{1}, 1, t_{3}\right)=\left(t_{3}-1\right) g_{n}\left(t_{1}, 1, t_{3}\right) .
$$

By the Torres formula (Lemma 2.3.),

$$
\Delta_{\bar{W}}\left(t_{1}, 1, t_{3}\right) \doteq\left(t_{3}-1\right) \Delta_{H_{1}}\left(t_{1}, t_{3}\right) \doteq t_{3}-1 .
$$

Hence we have $g_{n}\left(t_{1}, 1, t_{3}\right) \doteq 1$. Similarly we have $g_{n}\left(1, t_{2}, t_{3}\right) \doteq 1$.

We define an integer $\varepsilon_{n}$ by $-\varepsilon_{n}=g_{n}(1,1,1)$. Then $\varepsilon_{n}=1$ or -1 .

\section{Lemma 3.2.}

$$
g_{n}\left(t_{1}, 1,1\right)=-\varepsilon_{n} t_{1} \quad \text { and } \quad g_{n}\left(1, t_{2}, 1\right)=-\varepsilon_{n} t_{2} .
$$


Proof. By the duality of the Alexander polynomial (Lemma 2.4.), there exists integers $a, b$ and $c$ such that

$$
\Delta_{\bar{W}}\left(t_{1}, t_{2}, t_{3}\right)=-t_{1}^{a} t_{2}^{b} t_{3}^{c} \Delta_{\bar{W}}\left(t_{1}^{-1}, t_{2}^{-1}, t_{3}^{-1}\right)
$$

By substituting $t_{3}=1$ to (3.2), we have

$$
n\left(t_{1} t_{2}-1\right)\left(t_{1}-1\right)\left(t_{2}-1\right)=-n t_{1}^{a} t_{2}^{b}\left(t_{1}^{-1} t_{2}^{-1}-1\right)\left(t_{1}^{-1}-1\right)\left(t_{2}^{-1}-1\right)
$$

by (3.1). Then we have $a=b=2$. By (3.1) and (3.2), we have

$$
g_{n}\left(t_{1}, t_{2}, t_{3}\right)=t_{1}^{2} t_{2}^{2} t_{3}^{c-1} g_{n}\left(t_{1}^{-1}, t_{2}^{-1}, t_{3}^{-1}\right),
$$

and hence $g_{n}\left(t_{1}, 1,1\right)=t_{1}^{2} g_{n}\left(t_{1}^{-1}, 1,1\right)$. We then have the result by Lemma 3.1.

Let $E_{\bar{W}}$ be the exterior of $\bar{W}, m_{i}$ and $l_{i}$ a meridian and a longitude of $K_{i}(i=$ $1,2,3)$ on $\partial E_{\bar{W}}$ respectively, and set

$$
M=E_{\bar{W}} \cup V_{1} \cup V_{2} \cup V_{3} \text { and } \quad M_{0}=E_{\bar{W}} \cup V_{1} \cup V_{2},
$$

where $V_{i}$ is a solid torus glued in doing surgery along $K_{i}$. Let $m_{i}^{\prime}$ and $l_{i}^{\prime}$ be a meridian and a longitude of $V_{i}$ respectively. We may assume that, in $H_{1}\left(E_{\bar{W}}\right)$,

$$
\begin{gathered}
{\left[m_{i}^{\prime}\right]=\left[m_{i}\right]^{p_{i}}\left[l_{i}\right]^{q_{i}},\left[l_{i}^{\prime}\right]=\left[m_{i}\right]^{r_{i}}\left[l_{i}\right]^{s_{i}}, p_{i} s_{i}-q_{i} r_{i}=-1(i=1,2),} \\
{\left[m_{3}^{\prime}\right]=\left[m_{3}\right],\left[l_{3}^{\prime}\right]=\left[l_{3}\right],\left[l_{1}\right]=\left[l_{2}\right]=\left[m_{3}\right],\left[l_{3}\right]=\left[m_{1}\right]\left[m_{2}\right] .}
\end{gathered}
$$

Here [ - ] denotes the homology class in $H_{1}\left(E_{\bar{W}}\right)$. In the following, we also denote the homology class in $H_{1}\left(M_{0}\right)$ and $H_{1}(M)$ by the same symbol.

In $H_{1}\left(M_{0}\right)$, we have $\left[m_{i}^{\prime}\right]=\left[m_{i}\right]^{p_{i}}\left[l_{i}\right]^{q_{i}}=1(i=1,2)$. Hence we have

$$
H_{1}\left(M_{0}\right) \cong\left\langle\left[m_{1}\right],\left[m_{2}\right],\left[m_{3}\right] \mid\left[m_{i}\right]^{p_{i}}\left[m_{3}\right]^{q_{i}}=1(i=1,2)\right\rangle
$$

We set $T_{i}=\left[m_{i}\right]^{r_{i}}\left[m_{3}\right]^{s_{i}}(i=1,2)$. Then

$$
\begin{aligned}
{\left[m_{i}\right] } & =\left[m_{i}\right]^{-p_{i} s_{i}+q_{i} r_{i}} \\
& =\left(\left[m_{i}\right]^{p_{i}}\left[m_{3}\right]^{q_{i}}\right)^{-s_{i}}\left(\left[m_{i}\right]^{r_{i}}\left[m_{3}\right]^{s_{i}}\right)^{q_{i}}=T_{i}^{q_{i}}(i=1,2) \\
{\left[m_{3}\right] } & =\left[m_{3}\right]^{-p_{1} s_{1}+q_{1} r_{1}} \\
& =\left(\left[m_{1}\right]^{p_{1}}\left[m_{3}\right]^{q_{1}}\right)^{r_{1}}\left(\left[m_{1}\right]^{r_{1}}\left[m_{3}\right]^{s_{1}}\right)^{-p_{1}}=T_{1}^{-p_{1}}=T_{2}^{-p_{2}}
\end{aligned}
$$

By (3.3) and (3.4), we have

$$
H_{1}\left(M_{0}\right) \cong\left\langle T_{1}, T_{2} \mid T_{1}^{p_{1}}=T_{2}^{p_{2}}\right\rangle
$$

By the condition $\operatorname{gcd}\left(p_{1}, p_{2}\right)=1$, there exists integers $u_{1}, u_{2}$ such that $u_{2} p_{1}+u_{1} p_{2}=$ 1. We set $T=T_{1}^{u_{1}} T_{2}^{u_{2}}$. Then by (3.5), we have

$$
\begin{aligned}
& T_{1}=T_{1}^{u_{2} p_{1}+u_{1} p_{2}}=\left(T_{1}^{u_{1}} T_{2}^{u_{2}}\right)^{p_{2}}\left(T_{1}^{p_{1}} T_{2}^{-p_{2}}\right)^{u_{2}}=T^{p_{2}} \\
& T_{2}=T_{2}^{u_{2} p_{1}+u_{1} p_{2}}=\left(T_{1}^{u_{1}} T_{2}^{u_{2}}\right)^{p_{1}}\left(T_{1}^{p_{1}} T_{2}^{-p_{2}}\right)^{-u_{1}}=T^{p_{1}},
\end{aligned}
$$


and

$$
H_{1}\left(M_{0}\right) \cong\langle T \mid-\rangle \cong \mathbb{Z} .
$$

By (3.4) and (3.6), we have

(3.7) $\left[l_{i}^{\prime}\right]=T_{i}=T^{p_{i}} \neq 1 \quad(i=1,2)$

$\left[l_{3}\right]=\left[m_{1}\right]\left[m_{2}\right]=T_{1}^{q_{1}} T_{2}^{q_{2}}=T^{p_{2} q_{1}+p_{1} q_{2}} \neq 1$

in $H_{1}\left(M_{0}\right)$.

Let $\psi$ be as in the statement of Theorem 1.1. (1), and $\psi_{0}=\psi \circ \iota$ where $\iota$ : $\mathbb{Z}\left[H_{1}\left(M_{0}\right)\right] \rightarrow \mathbb{Z}\left[H_{1}(M)\right]$ is a ring homomorphism induced from the natural inclusion $M_{0} \hookrightarrow M$. Then by Lemma 2.1. (1), (3.1) and (3.7), we have

$$
\begin{aligned}
\tau\left(M_{0}\right) \doteq & \Delta_{\bar{W}}\left(T^{p_{2} q_{1}}, T^{p_{1} q_{2}}, T^{-p_{1} p_{2}}\right)\left(T^{p_{1}}-1\right)^{-1}\left(T^{p_{2}}-1\right)^{-1} \\
\doteq & \frac{n\left(T^{p_{2} q_{1}+p_{1} q_{2}}-1\right)\left(T^{p_{2} q_{1}}-1\right)\left(T^{p_{1} q_{2}}-1\right)}{\left(T^{p_{1}}-1\right)\left(T^{p_{2}}-1\right)} \\
& +\frac{\left(T^{-p_{1} p_{2}}-1\right)}{\left(T^{p_{1}}-1\right)\left(T^{p_{2}}-1\right)} g_{n}\left(T^{p_{2} q_{1}}, T^{p_{1} q_{2}}, T^{-p_{1} p_{2}}\right) \\
\doteq & n\left(T^{p_{2}\left(q_{1}-1\right)}+T^{p_{2}\left(q_{1}-2\right)}+\cdots+T^{p_{2}}+1\right) \\
& \cdot \frac{\left(T^{p_{2} q_{1}+p_{1} q_{2}}-1\right)\left(T^{p_{1} q_{2}}-1\right)}{T^{p_{1}}-1} \\
& -T^{-p_{1} p_{2}}\left(T^{p_{2}\left(p_{1}-1\right)}+T^{p_{2}\left(p_{1}-2\right)}+\cdots+T^{p_{2}}+1\right) \\
& . \frac{g_{n}\left(T^{p_{2} q_{1}}, T^{p_{1} q_{2}}, T^{-p_{1} p_{2}}\right)}{T^{p_{1}}-1} .
\end{aligned}
$$

Since $\left[m_{2}\right]=T^{p_{1} q_{2}}$ and $\psi_{0}\left(\left[m_{2}\right]\right)=\zeta_{d}$, we have $\psi_{0}(T)=\zeta_{d}^{\bar{p}_{1} \bar{q}_{2}}$ where $p_{1} \bar{p}_{1} \equiv$ $q_{2} \bar{q}_{2} \equiv 1(\bmod d)$. Hence we have

$$
\begin{aligned}
\tau^{\psi}(M) & \doteq\left\{\frac{n q_{1}\left(\zeta_{d}-1\right)^{2}}{\zeta_{d}^{\bar{q}_{2}}-1}-\frac{p_{1} g_{n}\left(1, \zeta_{d}, 1\right)}{\zeta_{d}^{\bar{q}_{2}}-1}\right\}\left(\zeta_{d}-1\right)^{-1} \\
& \doteq\left\{n q_{1}\left(\zeta_{d}-1\right)^{2}+\varepsilon_{n} p_{1} \zeta_{d}\right\}\left(\zeta_{d}-1\right)^{-1}\left(\zeta_{d}^{\bar{q}_{2}}-1\right)^{-1}
\end{aligned}
$$

by Lemma 2.1. (2) and Lemma 3.2.

Proof of (3). By computing the Alexander polynomial of $\bar{W}$ in Figure 3 for the case $n=1$, we have

$$
\begin{gathered}
g_{1}\left(t_{1}, t_{2}, t_{3}\right)=-\left(2 t_{1} t_{2}-t_{1}-t_{2}+1\right), \\
g_{1}\left(1, \zeta_{d}, 1\right)=-\zeta_{d},
\end{gathered}
$$


and $\varepsilon_{1}=1$.

Remark 3.3. We appreciate deeply that the referee computed

$$
g_{n}\left(t_{1}, t_{2}, t_{3}\right)=-n\left(t_{1}-1\right)\left(t_{2}-1\right)-t_{1} t_{2},
$$

and $\varepsilon_{n}=-g_{n}(1,1,1)=1$. To tell the truth, we have already recognized that it is not so defficult to calculate $g_{n}\left(t_{1}, t_{2}, t_{3}\right)$ as the referee pointed out. But we do not calculate it, because we do not need the explicit expression. The arguments in this section and the next section can be applied for more extended situations after some modifications. In Section 6, we will discuss about it (for the meaning of $\varepsilon_{n}$, see Remark 6.4.).

\section{Proof of "only if part" of Theorem 1.2.}

We will prove two lemmas: In Lemma 4.1., we will study the case $p_{1}$ (or $p_{2}$ ) is divisible by a prime $\ell \geq 5$. In Lemma 4.2., we will study the case $p_{1}$ (or $p_{2}$ ) is divisible by 2 or 3 . After that, we will prove "only if part" of Theorem 1.2. by the lemmas.

Lemma 4.1. Suppose that $M=\left(W_{n} ; p_{1} / q_{1}, p_{2} / q_{2}\right)$ is a lens space. Then we have the following:

(1) If $p_{2}$ is divisible by a prime $\ell \geq 5$, then we have $n=1, q_{1}=1$, and $p_{1}=1,2$ or 3 .

(2) If $p_{1}$ is divisible by a prime $\ell \geq 5$, then we have $n=1, q_{2}=1$, and $p_{2}=1,2$ or 3 .

Proof. We prove only the case (1). Suppose that $M=\left(W_{n} ; p_{1} / q_{1}, p_{2} / q_{2}\right)$ is a lens space.

By Theorem 1.1. and Example 2.2., there exists integers $i, j$ and $k$ with $\operatorname{gcd}(i, \ell)=\operatorname{gcd}(j, \ell)=\operatorname{gcd}(k, \ell)=1, k \equiv \pm \bar{q}_{2}(\bmod \ell)$,

$$
1 \leq i, j \leq \frac{\ell-1}{2}, \quad 1 \leq k \leq \ell-1 \quad \text { and } \quad i+j \equiv k+1(\bmod 2)
$$

such that

$$
\left\{n q_{1}\left(\zeta_{\ell}-1\right)^{2}+\varepsilon_{n} p_{1} \zeta_{\ell}\right\}\left(\zeta_{\ell}^{i}-1\right)\left(\zeta_{\ell}^{j}-1\right) \doteq\left(\zeta_{\ell}-1\right)\left(\zeta_{\ell}^{k}-1\right) .
$$

Case $1 i+j \equiv 1(\bmod 2)$.

Then the one of $i$ and $j$ is odd, and the other is even. By (4.1), $k$ is even, $3 \leq i+j \leq \ell-2$ and $3 \leq k+1 \leq \ell$. By (4.2), we have

$$
\zeta_{\ell}^{-\frac{i+j-1}{2}} \cdot\left\{n q_{1}\left(\zeta_{\ell}-1\right)^{2}+\varepsilon_{n} p_{1} \zeta_{\ell}\right\} \cdot \frac{\left(\zeta_{\ell}^{i}-1\right)\left(\zeta_{\ell}^{j}-1\right)}{\left(\zeta_{\ell}-1\right)\left(\zeta_{\ell}^{2}-1\right)}=\eta \zeta_{\ell}^{-\frac{k-2}{2}} \cdot \frac{\zeta_{\ell}^{k}-1}{\zeta_{\ell}^{2}-1} \in \mathbb{R}
$$


where $\eta= \pm 1$. By Lemma 2.6., we have

$$
t^{-\frac{i+j-1}{2}} \cdot\left\{n q_{1}(t-1)^{2}+\varepsilon_{n} p_{1} t\right\} \cdot \frac{\left(t^{i}-1\right)\left(t^{j}-1\right)}{(t-1)\left(t^{2}-1\right)}=\eta t^{-\frac{k-2}{2}} \cdot \frac{t^{k}-1}{t^{2}-1} \in \mathbb{Z}\left[t, t^{-1}\right]
$$

Hence we have $n=1$ and $q_{1}=1$. Thus $(t-1)^{2}+p_{1} t$ is a divisor of $t^{k}-1$, and hence it is the third, fourth or sixth cyclotomic polynomial:

$$
(t-1)^{2}+\varepsilon_{1} p_{1} t=t^{2}+t+1, \quad t^{2}+1 \quad \text { or } t^{2}-t+1 .
$$

Hence we have $p_{1}=\varepsilon_{1}, 2 \varepsilon_{1}$ or $3 \varepsilon_{1}$. Recall that $\varepsilon_{1}=1$ (Theorem 1.1. (3)). Therefore we have $p_{1}=1,2$ or 3 .

Case $2 i+j \equiv 0(\bmod 2)$.

Then by (4.1), $k$ is odd, $2 \leq i+j \leq \ell-1$ and $2 \leq k+1 \leq \ell-1$. By (4.2), we have

$$
\zeta_{\ell}^{-\frac{i+j}{2}} \cdot\left\{n q_{1}\left(\zeta_{\ell}-1\right)^{2}+\varepsilon_{n} p_{1} \zeta_{\ell}\right\} \cdot \frac{\left(\zeta_{\ell}^{i}-1\right)\left(\zeta_{\ell}^{j}-1\right)}{\left(\zeta_{\ell}-1\right)^{2}}=\eta \zeta_{\ell}^{-\frac{k-1}{2}} \cdot \frac{\zeta_{\ell}^{k}-1}{\zeta_{\ell}-1} \in \mathbb{R}
$$

where $\eta= \pm 1$. Suppose that $(i, j) \neq\left(\frac{\ell-1}{2}, \frac{\ell-1}{2}\right)$. Then we have

$$
t^{-\frac{i+j}{2}} \cdot\left\{n q_{1}(t-1)^{2}+\varepsilon_{n} p_{1} t\right\} \cdot \frac{\left(t^{i}-1\right)\left(t^{j}-1\right)}{(t-1)^{2}}=\eta t^{-\frac{k-1}{2}} \cdot \frac{t^{k}-1}{t-1} \in \mathbb{Z}\left[t, t^{-1}\right]
$$

by Lemma 2.6.. As in Case 1, we have the result.

Suppose that $i=j=\frac{\ell-1}{2}$. We set

$$
\begin{aligned}
& A=\zeta_{\ell}^{-\frac{\ell+1}{2}} \cdot\left\{n q_{1}\left(\zeta_{\ell}-1\right)^{2}+\varepsilon_{n} p_{1} \zeta_{\ell}\right\}\left(\zeta_{\ell}^{\frac{\ell-1}{2}}-1\right)^{2}, \\
& B=\zeta_{\ell}^{-\frac{k+1}{2}} \cdot\left(\zeta_{\ell}-1\right)\left(\zeta_{\ell}^{k}-1\right) .
\end{aligned}
$$

Then $A=\eta B$ holds. By expanding $A$ and $B$, we have

$$
\begin{aligned}
A= & -2\left(\varepsilon_{n} p_{1}-2 n q_{1}\right)-2 n q_{1}\left(\zeta_{\ell}+\zeta_{\ell}^{-1}\right)+n q_{1}\left(\zeta_{\ell}^{\frac{\ell-3}{2}}+\zeta_{\ell}^{-\frac{\ell-3}{2}}\right) \\
& +\left(\varepsilon_{n} p_{1}-n q_{1}\right)\left(\zeta_{\ell}^{\frac{\ell-1}{2}}+\zeta_{\ell}^{-\frac{\ell-1}{2}}\right), \\
B= & -\left(\zeta_{\ell}^{\frac{k-1}{2}}+\zeta_{\ell}^{-\frac{k-1}{2}}\right)+\left(\zeta_{\ell}^{\frac{k+1}{2}}+\zeta_{\ell}^{-\frac{k+1}{2}}\right) .
\end{aligned}
$$

If $\ell \geq 7$, then

$$
\begin{aligned}
A= & -\left(3 \varepsilon_{n} p_{1}-5 n q_{1}\right)-\left(\varepsilon_{n} p_{1}+n q_{1}\right)\left(\zeta_{\ell}+\zeta_{\ell}^{-1}\right) \\
& -\left(\varepsilon_{n} p_{1}-n q_{1}\right) \sum_{i=2}^{\frac{\ell-5}{2}}\left(\zeta_{\ell}^{i}+\zeta_{\ell}^{-i}\right)-\left(\varepsilon_{n} p_{1}-2 n q_{1}\right)\left(\zeta_{\ell}^{\frac{\ell-3}{2}}+\zeta_{\ell}^{-\frac{\ell-3}{2}}\right) .
\end{aligned}
$$

By Lemma 2.6., we have the following: 
(i) If $k=1$, then no $n, p_{1}$ and $q_{1}$ satisfy $A=\eta B$.

(ii) If $3 \leq k \leq \ell-4$, then no $n, p_{1}$ and $q_{1}$ satisfy $A=\eta B$.

(iii) If $k=\ell-2$, then we have

$$
B=-1-\sum_{i=1}^{\frac{\ell-5}{2}}\left(\zeta_{\ell}^{i}+\zeta_{\ell}^{-i}\right)-2\left(\zeta_{\ell}^{\frac{\ell-3}{2}}+\zeta_{\ell}^{-\frac{\ell-3}{2}}\right)
$$

and hence no $n, p_{1}$ and $q_{1}$ satisfy $A=\eta B$.

If $\ell=5$, then we have

$$
\begin{aligned}
A & =-2\left(\varepsilon_{n} p_{1}-2 n q_{1}\right)-n q_{1}\left(\zeta_{\ell}+\zeta_{\ell}^{-1}\right)+\left(\varepsilon_{n} p_{1}-n q_{1}\right)\left(\zeta_{\ell}^{2}+\zeta_{\ell}^{-2}\right) \\
& =-\left(3 \varepsilon_{n} p_{1}-5 n q_{1}\right)-\varepsilon_{n} p_{1}\left(\zeta_{\ell}+\zeta_{\ell}^{-1}\right) .
\end{aligned}
$$

(i) If $k=1$, then we have $B=-2+\left(\zeta_{\ell}+\zeta_{\ell}^{-1}\right)$, and hence we have $n=p_{1}=$ $q_{1}=1$.

(ii) If $k=3$, then we have $B=-1-2\left(\zeta_{\ell}+\zeta_{\ell}^{-1}\right)$, and hence we have $n=1$, $p_{1}=2$ and $q_{1}=1$.

Therefore this completes the proof.

Lemma 4.2. Suppose that $M=\left(W_{n} ; p_{1} / q_{1}, p_{2} / q_{2}\right)$ is a lens space. Then we have:

(1) If $n=1$ and $p_{1} / q_{1}=1$, then we have $\left|p_{2}-6 q_{2}\right|=1$.

(2) If $p_{1}$ is divisible by 2 , then we have $\left|\varepsilon_{n} p_{2}-4 n q_{2}\right|=1$.

(3) If $p_{1}$ is divisible by 3 , then we have $\left|\varepsilon_{n} p_{2}-3 n q_{2}\right|=1$.

(4) If $p_{1}$ is divisible by 4 , then we have $\left|\varepsilon_{n} p_{2}-2 n q_{2}\right|=1$.

(5) If $n=1$ and $p_{2} / q_{2}=1$, then we have $\left|p_{1}-6 q_{1}\right|=1$.

(6) If $p_{2}$ is divisible by 2 , then we have $\left|\varepsilon_{n} p_{1}-4 n q_{1}\right|=1$.

(7) If $p_{2}$ is divisible by 3 , then we have $\left|\varepsilon_{n} p_{1}-3 n q_{1}\right|=1$.

(8) If $p_{2}$ is divisible by 4 , then we have $\left|\varepsilon_{n} p_{1}-2 n q_{1}\right|=1$.

Proof. (1) If $n=1$ and $p_{1} / q_{1}=1$, then $M$ is the result of $p_{2} / q_{2}$-surgery along the $(2,3)$-torus knot (i.e. the righthand trefoil). Hence we have $\left|p_{2}-6 q_{2}\right|=1$ by the result of L. Moser [17], and then $M=L\left(p_{2}, 4 q_{2}\right)$. The case (5) is similarly shown.

We prove only (6), (7) and (8).

(6) Suppose that $p_{2}$ is divisible by 2 . Since $\zeta_{2}=-1$, and $i, j$ and $k$ are odd in (4.2), we have

$$
n q_{1}(-1-1)^{2}+\varepsilon_{n} p_{1}(-1)=4 n q_{1}-\varepsilon_{n} p_{1}= \pm 1
$$

and $\left|\varepsilon_{n} p_{1}-4 n q_{1}\right|=1$ by (4.2). The case (2) is similarly shown. 
(7) Suppose that $p_{2}$ is divisible by 3. Since $\left|\zeta_{3}-1\right|=\left|\zeta_{3}^{i}-1\right|=\left|\zeta_{3}^{j}-1\right|=\left|\zeta_{3}^{k}-1\right| \neq 0$, and

$$
n q_{1}\left(\zeta_{3}-1\right)^{2}+\varepsilon_{n} p_{1} \zeta_{3}=\zeta_{3}\left(\varepsilon_{n} p_{1}-3 n q_{1}\right),
$$

we have $\left|\varepsilon_{n} p_{1}-3 n q_{1}\right|=1$ by (4.2). The case (3) is similarly shown.

(8) Suppose that $p_{2}$ is divisible by 4 . Since $\left|\zeta_{4}-1\right|=\left|\zeta_{4}^{i}-1\right|=\left|\zeta_{4}^{j}-1\right|=\left|\zeta_{4}^{k}-1\right| \neq 0$, and

$$
n q_{1}\left(\zeta_{4}-1\right)^{2}+\varepsilon_{n} p_{1} \zeta_{4}=\zeta_{4}\left(\varepsilon_{n} p_{1}-2 n q_{1}\right),
$$

we have $\left|\varepsilon_{n} p_{1}-2 n q_{1}\right|=1$ by (4.2). The case (4) is similarly shown. This completes the proof.

Proof of the "only if part" of Theorem 1.2. By Lemma 4.2., it is sufficient to prove that $n=1$, and at least one of $p_{1} / q_{1}$ and $p_{2} / q_{2}$ is 1,2 or 3 .

Case 1 At least one of $p_{1}$ and $p_{2}$ has a prime divisor $\ell \geq 5$.

Suppose that $p_{2}$ has a prime divisor $\ell \geq 5$. By Lemma 4.1. (1), we have $n=1$, and $p_{1} / q_{1}=1,2$ or 3 . The case that $p_{1}$ has a prime divisor $\ell \geq 5$ is similarly shown.

Case 2 Otherwise, i.e. both $\left|p_{1}\right|$ and $\left|p_{2}\right|$ are of type $2^{a} 3^{b}(a, b \in \mathbb{Z} ; a \geq 0, b \geq 0)$.

Recall that $p_{1}$ and $p_{2}$ are coprime.

Case 2-1 Either $p_{1}$ or $p_{2}$ is divisible by 6 .

Suppose that $p_{2}$ is divisible by 6 . Then we have $p_{1}= \pm 1$ by coprimeness. This case does not occur by Lemma 4.2. (6) or (7). The case that $p_{1}$ is divisible by 6 is similarly shown.

Case 2-2 Either $p_{1}$ or $p_{2}$ is divisible by 4 .

Suppose that $p_{2}$ is divisible by 4. By Lemma 4.2. (6) and (8), we have $n=1$, $q_{1}=1$ and $\varepsilon_{n} p_{1}=3$. By Theorem 1.1. (3), we have $p_{1} / q_{1}=3$. The case that $p_{1}$ is divisible by 4 is similarly shown.

Case 2-3 $\left\{\left|p_{1}\right|,\left|p_{2}\right|\right\}=\left\{1,3^{b}\right\}$ or $\left\{2,3^{b}\right\}$.

Suppose that $\left|p_{1}\right|=1$ or 2 , and $\left|p_{2}\right|=3^{b}$. By Lemma 4.2. (7), we have $n=1$, $q_{1}=1$ and $\varepsilon_{n} p_{1}=2$. By Theorem 1.1. (3), we have $p_{1} / q_{1}=2$. The case that $\left|p_{2}\right|=1$ or 2 , and $\left|p_{1}\right|=3^{b}$ is similarly shown.

Case 2-4 $\left\{\left|p_{1}\right|,\left|p_{2}\right|\right\}=\{1,2\}$.

By Lemma 4.2. (6), these cases do not occur.

\section{Proof of "if part" of Theorem 1.2.}

We need the following fact proved in [10].

Lemma 5.1. Let $L$ be a $(2,2 s)$-torus link where $|s| \geq 2$, and $M=\left(L ; \alpha_{1} / \beta_{1}\right.$, $\left.\alpha_{2} / \beta_{2}\right)$ the result of Dehn surgery along $L$ where $\alpha_{i}$ and $\beta_{i}(i=1,2)$ are integers 
such that $\left|\alpha_{i}-s \beta_{i}\right| \neq 0$. Then $M$ is a lens space if and only if $\left|\alpha_{1}-s \beta_{1}\right|=1$ or $\left|\alpha_{2}-s \beta_{2}\right|=1$. Moreover if $\left|\alpha_{2}-s \beta_{2}\right|=1$, then $M=L\left(p,\left(\alpha_{1}-s \beta_{1}\right) \beta_{2}+\varepsilon \beta_{1}\right)$ where $p=\alpha_{1} \alpha_{2}-s^{2} \beta_{1} \beta_{2}$ and $\varepsilon=\alpha_{2}-s \beta_{2}(= \pm 1)$.

Proof of the "if part" of Theorem 1.2.

(a) The case $p_{1} / q_{1}=1$, or $p_{2} / q_{2}=1$.

We have already shown the lens surgery in the proof of Lemma 4.2. (1).

(b) The case $p_{1} / q_{1}=2$, or $p_{2} / q_{2}=2$.

We prove only the case $p_{1} / q_{1}=2$. We have a framed link presentation of $M$ as in Figure 4 which is Dehn surgery along a $(2,4)$-torus link where we set $r=p_{2} / q_{2}$. Since this case is $s=2, \alpha_{1}=-2, \beta_{1}=1, \alpha_{2}=p_{2}-2 q_{2}$ and $\beta_{2}=q_{2}$ in Lemma 5.1., $M$ is a lens space if and only if $\left|\left(p_{2}-2 q_{2}\right)-2 q_{2}\right|=\left|p_{2}-4 q_{2}\right|=1$, and then $M=L\left(2 p_{2}, 8 q_{2}-p_{2}\right)$.

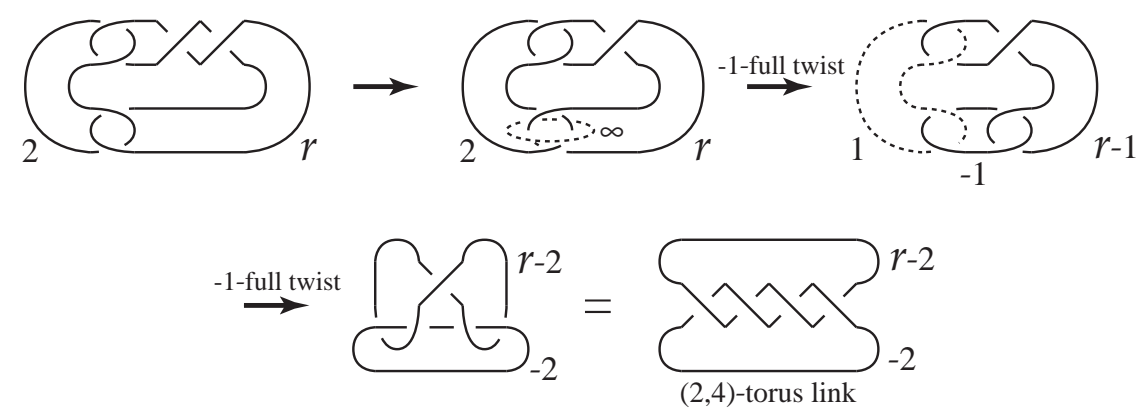

Figure 4: $(2, r)$-surgery along $W_{1}$

(c) The case $p_{1} / q_{1}=3$, or $p_{2} / q_{2}=3$.

We prove only the case $p_{1} / q_{1}=3$. We have a framed link presentation of $M$ as in Figure 5 which is Dehn surgery along a $(2,-6)$-torus link where we set $r=p_{2} / q_{2}$. Since this case is $s=-3, \alpha_{1}=-3, \beta_{1}=2, \alpha_{2}=p_{2}-6 q_{2}$ and $\beta_{2}=q_{2}$ in Lemma 5.1., $M$ is a lens space if and only if $\left|\left(p_{2}-6 q_{2}\right)+3 q_{2}\right|=\left|p_{2}-3 q_{2}\right|=1$, and then $M=L\left(3 p_{2}, 3 q_{2}-2 p_{2}\right)$. The case $p_{2} / q_{2}=3$ is similarly shown.

Therefore this completes the proof.

\section{Generalization of Theorem 1.2 .}

Our method extends to algebraically same links with $W_{n}$. Let $L=K_{1} \cup K_{2}$ be a 2 -component link in an integral homology 3 -sphere $\Sigma$ with its Alexander polynomials

$$
\Delta_{L}\left(t_{1}, t_{2}\right)=n\left(t_{1}-1\right)\left(t_{2}-1\right)(n \geq 0), \Delta_{K_{1}}(t) \doteq 1 \text { and } \Delta_{K_{2}}(t) \doteq 1 \text {. }
$$

Since we can take a 3 -ball $B$ in $\Sigma$ such that $B \cap K_{i} \neq \emptyset(i=1,2)$ and $(B, B \cap L)$ is a trivial 2-string tangle, we can add the third component $K_{3}$ in $B$ such that 


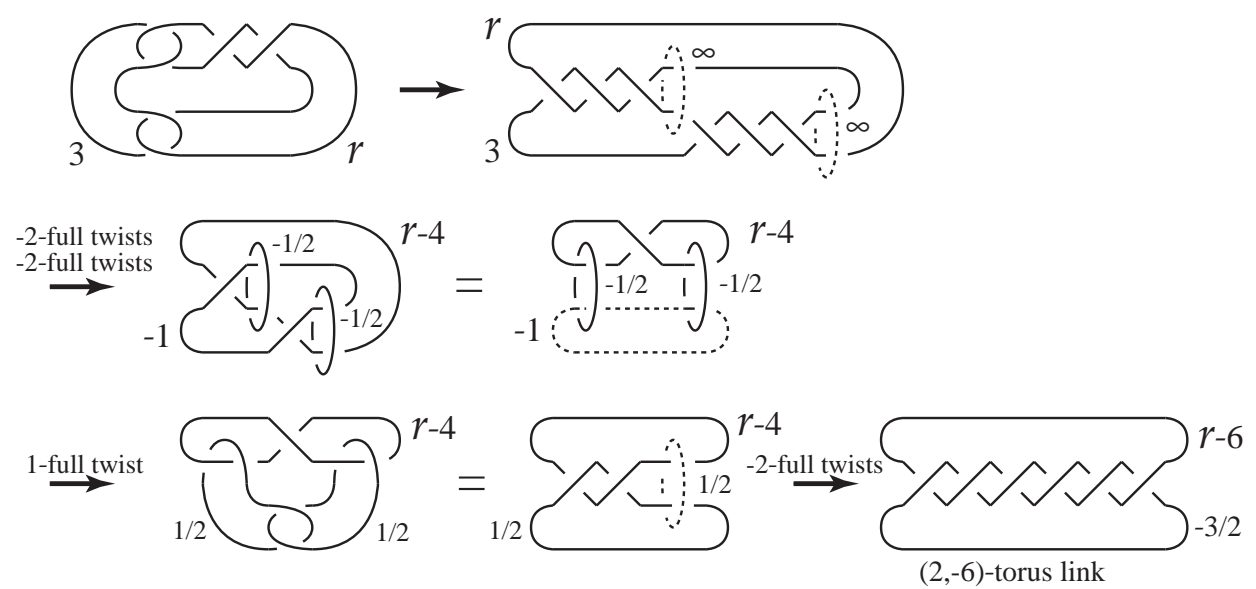

Figure 5: $(3, r)$-surgery along $W_{1}$

$H_{i}=K_{i} \cup K_{3}(i=1,2)$ is the connected sum of $K_{i}$ and the Hopf link, and $\operatorname{lk}\left(K_{i}, K_{3}\right)=1(i=1,2)$ by suitable orientations. We set $\bar{L}=L \cup K_{3}$. Then by the surgery formula (Lemma 2.1.) and (6.1), we have

$$
\Delta_{H_{i}}\left(t_{1}, t_{2}\right) \doteq \Delta_{K_{i}}\left(t_{i}\right) \doteq 1(i=1,2),
$$

and by the Torres formula (Lemma 2.3.) and (6.1), we may set as follows:

$$
\Delta_{\bar{L}}\left(t_{1}, t_{2}, t_{3}\right)=n\left(t_{1} t_{2}-1\right)\left(t_{1}-1\right)\left(t_{2}-1\right)+\left(t_{3}-1\right) g_{n}\left(t_{1}, t_{2}, t_{3}\right)
$$

for some $g_{n}\left(t_{1}, t_{2}, t_{3}\right) \in \mathbb{Z}\left[t_{1}^{ \pm 1}, t_{2}^{ \pm 1}, t_{3}^{ \pm 1}\right]$, which is just the same form as (3.1). We define an integer $\varepsilon_{n}$ by $-\varepsilon_{n}=g_{n}(1,1,1)$. Then for the case $n>0$, the same arguments as in Section 3 and Section 4 work by replacing $W_{n}$ and $\bar{W}$ with $L$ and $\bar{L}$, respectively, except the parts corresponding to Lemma 4.2. (1) and (5). In particular, Lemma 3.2. also holds for the case $n>0$ in the present setting.

Lemma 6.1. In the situation above, if $n>0$, then $\varepsilon_{n}=1$ or -1 is uniquely determined (i.e. $\varepsilon_{n}$ is well-defined), and $\left|\varepsilon_{0}\right|=1$.

Proof. Since Lemma 3.1. also holds by replacing $\bar{W}$ with $\bar{L}$, we have $\left|\varepsilon_{n}\right|=1$ for every $n$ including the case $n=0$. We show uniqueness of $\varepsilon_{n}$ for the case $n>0$. Let $M=(\bar{L} ; \emptyset, 1, \infty), M_{0}=(\bar{L} ; \emptyset, 1, \emptyset), E_{\bar{L}}$ the exterior of $\bar{L}, m_{i}$ and $l_{i}$ a meridian and a longitude of $K_{i}(i=1,2,3)$ on $\partial E_{\bar{L}}$ respectively, and set

$$
M=E_{\bar{L}} \cup V_{2} \cup V_{3} \quad \text { and } \quad M_{0}=E_{\bar{L}} \cup V_{2},
$$

where $V_{i}$ is a solid torus glued in doing surgery along $K_{i}$. Let $m_{i}^{\prime}$ and $l_{i}^{\prime}$ be a 
meridian and a longitude of $V_{i}$ respectively. We may assume that, in $H_{1}\left(E_{\bar{L}}\right)$,

$$
\begin{gathered}
{\left[m_{2}^{\prime}\right]=\left[m_{2}\right]\left[l_{2}\right],\left[l_{2}^{\prime}\right]=\left[m_{2}\right],\left[m_{3}^{\prime}\right]=\left[m_{3}\right],\left[l_{3}^{\prime}\right]=\left[l_{3}\right],} \\
{\left[l_{1}\right]=\left[l_{2}\right]=\left[m_{3}\right],\left[l_{3}\right]=\left[m_{1}\right]\left[m_{2}\right] .}
\end{gathered}
$$

Here [ - ] denotes the homology class in $H_{1}\left(E_{\bar{L}}\right)$. In the following, we also denote the homology class in $H_{1}\left(M_{0}\right)$ and $H_{1}(M)$ by the same symbol.

In $H_{1}\left(M_{0}\right)$, we have $\left[m_{2}^{\prime}\right]=\left[m_{2}\right]\left[l_{2}\right]=\left[m_{2}\right]\left[m_{3}\right]=1$. Hence we have

$$
H_{1}\left(M_{0}\right) \cong\left\langle\left[m_{1}\right],\left[m_{2}\right],\left[m_{3}\right] \mid\left[m_{2}\right]\left[m_{3}\right]=1\right\rangle \cong\left\langle\left[m_{1}\right],\left[m_{2}\right] \mid-\right\rangle \cong \mathbb{Z}^{2} .
$$

Then by the surgery formula (Lemma 2.1.) and (6.2), we have

$$
\begin{aligned}
\tau\left(M_{0}\right) & \doteq \Delta_{\bar{L}}\left(t_{1}, t_{2}, t_{2}^{-1}\right)\left(t_{2}-1\right)^{-1} \\
& \doteq n\left(t_{1} t_{2}-1\right)\left(t_{1}-1\right)-t_{2}^{-1} g_{n}\left(t_{1}, t_{2}, t_{2}^{-1}\right)
\end{aligned}
$$

In $H_{1}(M)$, we have $\left[m_{3}^{\prime}\right]=\left[m_{3}\right]=1$. Hence we have

$$
H_{1}(M) \cong\left\langle\left[m_{1}\right],\left[m_{2}\right],\left[m_{3}\right] \mid\left[m_{2}\right]=\left[m_{3}\right]=1\right\rangle \cong\left\langle\left[m_{1}\right] \mid-\right\rangle \cong \mathbb{Z} .
$$

Then by the surgery formula (Lemma 2.1.), (6.3) and Lemma 3.2., we have

$$
\begin{aligned}
\tau(M) & \doteq\left\{n\left(t_{1}-1\right)^{2}-g_{n}\left(t_{1}, 1,1\right)\right\}\left(t_{1}-1\right)^{-1} \\
& \doteq\left\{n\left(t_{1}-1\right)^{2}+\varepsilon_{n} t_{1}\right\}\left(t_{1}-1\right)^{-1} .
\end{aligned}
$$

Since $\tau(M)$ depends only on $L$ (i.e. independent from the third component $K_{3}$ ), and characterizes $\varepsilon_{n}, \varepsilon_{n}$ is uniquely determined as an invariant of $L$.

We remark that if $n=0$, then we cannot determine $\varepsilon_{0}$ uniquely. The value $\varepsilon_{n}$ for $n>0$ depends on the geometric shape of $L$ (see Remark 6.4.).

Since the first term of the righthand side of (6.2) vanishes for the case $n=0$, we may also assume Lemma 3.2 for $n=0$. Hence Theorem 1.1. (1) and (2) also hold for $n \geq 0$. Computations of the Reidemeister torsions in the present setting is the same as that in Section 3 and Section 4 by replacing $\bar{W}$ with $\bar{L}$. Then we have an extension of Lemma 4.1.

Theorem 6.2. Suppose that $M=\left(L ; p_{1} / q_{1}, p_{2} / q_{2}\right)$ is a lens space. Then we have the following:

(1) $n=0$ or 1 .

(2) If $n=0$, then $\left|p_{1}\right|=1$ or $\left|p_{2}\right|=1$. Moreover if $\left|p_{1}\right|=1$, then $M=$ $L\left(p_{2}, \pm q_{2}\right)$.

(3) If $n=1$ and $\left|p_{2}\right| \geq 5$, then $q_{1}=1$ and $p_{1}=\varepsilon_{1}, 2 \varepsilon_{1}$ or $3 \varepsilon_{1}$.

(4) If $n=1$ and $\left|p_{1}\right| \geq 5$, then $q_{2}=1$ and $p_{2}=\varepsilon_{1}, 2 \varepsilon_{1}$ or $3 \varepsilon_{1}$. 
In each case (3) and (4), $\varepsilon_{1}=1$ or -1 which is determined uniquely depending on $L$.

Proof. Firstly, we suppose $n>0$. Since the arguments in the proof of Lemma 4.1. also work in the present setting, we have $n=1$, and (3) and (4). Secondly, we suppose $n=0$. Then by the value of the Reidemeister torsion, we have (2).

An extensions of Theorem 1.2. (Lemma 4.2.) can also be obtained (cf. [5] for (1) and (4)).

Theorem 6.3. Suppose that $n=1$ and $M=\left(L ; p_{1} / q_{1}, p_{2} / q_{2}\right)$ is a lens space. Then one of the following (1), (2), (3), (4), (5) or (6) holds:

(1) $p_{1} / q_{1}=\varepsilon_{1}, \operatorname{gcd}\left(p_{2}, 6\right)=1$ and $6 q_{2} \equiv \pm 1\left(\bmod p_{2}\right)$.

(2) $p_{1} / q_{1}=2 \varepsilon_{1}$ and $\left|\varepsilon_{1} p_{2}-4 q_{2}\right|=1$.

(3) $p_{1} / q_{1}=3 \varepsilon_{1}$ and $\left|\varepsilon_{1} p_{2}-3 q_{2}\right|=1$.

(4) $p_{2} / q_{2}=\varepsilon_{1}, \operatorname{gcd}\left(p_{1}, 6\right)=1$ and $6 q_{1} \equiv \pm 1\left(\bmod p_{1}\right)$.

(5) $p_{2} / q_{2}=2 \varepsilon_{1}$ and $\left|\varepsilon_{1} p_{1}-4 q_{1}\right|=1$.

(6) $p_{2} / q_{2}=3 \varepsilon_{1}$ and $\left|\varepsilon_{1} p_{1}-3 q_{1}\right|=1$.

In each case (1), (2), (3), (4), (5) and (6), $\varepsilon_{1}=1$ or -1 which is determined uniquely depending on $L$.

Proof. Since the arguments in the proof of Lemma 4.2. also work in the present setting, we have (2), (3), (5) and (6). By the values of the Reidemeister torsions, we have (1) and (4).

Remark 6.4. The number $\varepsilon_{n}$ may be understood from several viewpoints. We remark here one of them. The forms of the Reidemeister torsions in Theorem 1.1. show that both $W_{n}$ and $L$ in this section for $n>0$ are not amphicheiral. For the case of $W_{1}$, Theorem 1.2. shows its chirality more clearly. They motivate a work of the first author [8] on the conditions for the Alexander polynomials of algebraically split component-preservingly amphicheiral links.

Let $\bar{L}(n \geq 0)$ be an oriented 3-component link in this section which is also expressed as $(\Sigma, \bar{L})$. We set its mirror imaged manifold pair as $\left(\Sigma^{\prime}, \overline{L^{\prime}}\right)=(\Sigma, \bar{L})$ ! where $\Sigma^{\prime}$ is the orientation-reversed $\Sigma$, and $\overline{L^{\prime}}=K_{1}^{\prime} \cup K_{2}^{\prime} \cup K_{3}^{\prime}$ has the induced orientation from $\bar{L}$, and set the 2-component sublink of $\bar{L}^{\prime}$ corrsponding to $L$ as $L^{\prime}=K_{1}^{\prime} \cup K_{2}^{\prime}\left(K_{i}^{\prime}(i=1,2,3)\right.$ corresponds to $\left.K_{i}\right)$. Then the Alexander polynomial of $\overline{L^{\prime}}$ is the same as that of $\bar{L}$ (up to trivial units). Though $\overline{L^{\prime}}$ looks satisfying the same conditions as $\bar{L}$, only $\mathrm{lk}\left(K_{i}^{\prime}, K_{3}^{\prime}\right)=-1(i=1,2)$ is different. Thus we re-set as $\overline{L^{\prime}}=K_{1}^{\prime} \cup K_{2}^{\prime} \cup\left(-K_{3}^{\prime}\right)$ where $\left(-K_{3}^{\prime}\right)$ is the orientation-reversed component of $K_{3}^{\prime}$. The Alexander polynomials of both $\bar{L}$ and $\overline{L^{\prime}}$ satisfy (6.2) where we set the 
$g_{n}\left(t_{1}, t_{2}, t_{3}\right)$-part for $\overline{L^{\prime}}$ as $g_{n}^{\prime}\left(t_{1}, t_{2}, t_{3}\right) \in \mathbb{Z}\left[t_{1}^{ \pm 1}, t_{2}^{ \pm 1}, t_{3}^{ \pm 1}\right]$. Then we have

$$
\begin{aligned}
\Delta_{\overline{L^{\prime}}}\left(t_{1}, t_{2}, t_{3}\right) & \doteq \Delta_{\bar{L}}\left(t_{1}, t_{2}, t_{3}^{-1}\right) \\
& \doteq n\left(t_{1} t_{2}-1\right)\left(t_{1}-1\right)\left(t_{2}-1\right)+\left(t_{3}^{-1}-1\right) g_{n}\left(t_{1}, t_{2}, t_{3}^{-1}\right) \\
& \doteq n\left(t_{1} t_{2}-1\right)\left(t_{1}-1\right)\left(t_{2}-1\right)+\left(t_{3}-1\right)\left(-t_{3}^{-1}\right) g_{n}\left(t_{1}, t_{2}, t_{3}^{-1}\right) \\
& \doteq n\left(t_{1} t_{2}-1\right)\left(t_{1}-1\right)\left(t_{2}-1\right)+\left(t_{3}-1\right) g_{n}^{\prime}\left(t_{1}, t_{2}, t_{3}\right) .
\end{aligned}
$$

We define an integer $\varepsilon_{n}$ by $-\varepsilon_{n}=g_{n}(1,1,1)$. Since we can take $g_{n}^{\prime}\left(t_{1}, t_{2}, t_{3}\right)=$ $-t_{3}^{-1} g_{n}\left(t_{1}, t_{2}, t_{3}^{-1}\right)$ and Lemma 6.1., we have $\varepsilon_{n}^{\prime}=-\varepsilon_{n}$ for $n>0$. Therefore $L$ cannot be amphicheiral in this case (i.e. only the case $n=0$ can be amphicheiral), and the statements of Theorem 6.2. and Theorem 6.3. have symmetries of this kind. In [8], it is conjectured that the Alexander polynomial of an algebraically split component-preservingly amphicheiral link with even components is zero.

\section{References}

[1] J. Berge, Some knots with surgeries yielding lens spaces, (Unpublished manuscript, 1990).

[2] M. Culler, M. Gordon, J. Luecke and P. Shalen, Dehn surgery on knots, Ann. of Math., 125(1987), 237-300.

[3] R. Fintushel and R. J. Stern, Constructing lens spaces by surgery on knots, Math. Z., 175(1980), no.1, 33-51.

[4] H. Goda and M. Teragaito, Dehn surgeries on knots which yield lens spaces and genera of knots, Math. Proc. Cambridge Philos. Soc., 129(2000), No.3 , 501-515.

[5] T. Kadokami, Reidemeister torsion and lens surgeries on knots in homology 3-spheres I, Osaka J. Math., 43(2006), no.4, 823-837.

[6] T. Kadokami, Reidemeister torsion of Seifert fibered homology lens spaces and Dehn surgery, Algebr. Geom. Topol., 7(2007), 1509-1529.

[7] T. Kadokami, Reidemeister torsion and lens surgeries on knots in homology 3-spheres II, Top. Appl., 155(2008), no.15 , 1699-1707.

[8] T. Kadokami, Amphicheiral links with special properties, I, to appear in Jounal of Knot Theory and its Ramifications.

[9] T. Kadokami, Finite slope surgeries along the Milnor links, in preparation.

[10] T. Kadokami and M. Shimozawa, Dehn surgery along torus links, J. Knot Theory Ramif., 19(2010), 489-502.

[11] T. Kadokami and Y. Yamada, Reidemeister torsion and lens surgeries on $(-2, m, n)$ pretzel knots, Kobe J. Math., 23(2006), 65-78.

[12] T. Kadokami and Y. Yamada, A deformation of the Alexander polynomials of knots yielding lens spaces, Bull. of Austral. Math. Soc., 75(2007), 75-89.

[13] A. Kawauchi, A survey of Knot Theory, Birkhäuser Verlag, (1996). 
[14] R. Kirby, A calculus for framed links in $S^{3}$, Invent. Math., 45(1978), no.1, 35-56.

[15] B. Martelli and C. Petronio, Dehn filling of the "magic" 3-manifold, Comm. Anal. Geom., 14(2006), No. 5, 969-1026.

[16] N. Maruyama, On Dehn surgery along a certain family of knots, Jour. of Tsuda College, 19(1987), 261-280.

[17] L. Moser, Elementary surgery along a torus knot, Pacific J. Math., 38(1971), 737-745.

[18] P. Ozsváth and Z. Szabó, On knot Floer homology and lens space surgeries, Topology, 44(2005), 1281-1300.

[19] K. Reidemeister, Homotopieringe und Linsenräume, Abh. Math. Sem. Univ. Hamburg, 11(1935), 102-109.

[20] D. Rolfsen, Rational surgery calculus: extension of Kirby's theorem, Pacific J. Math., 110(1984), 377-386.

[21] G. Torres, On the Alexander polynomial, Ann. of Math., 57(1953), 57-89.

[22] V. G. Turaev, Reidemeister torsion in knot theory, Russian Math. Surveys, 411(1986), 119-182.

[23] V. G. Turaev, Introduction to Combinatorial Torsions, Birkhäuser Verlag, (2001). 